Markus Pausch

\title{
Nur weil die Welt absurd ist, brauchen wir eine Demokratie
}

\author{
Politiktheoretische Anknüpfungspunkte im Werk Albert Camus'
}

\section{Einleitung}

Als der deutsche Sozialdemokrat Günter Bartsch im Jahre 1965 in der Theoriezeitschrift »Die Neue Gesellschaft « einen Artikel zum Thema »Camus und der demokratische Sozialismus « schrieb, war Albert Camus bereits seit fünf Jahren tot. »Es ist seltsam und befremdend, wie schnell die politischen Aspekte des Werkes von Albert Camus, der am 5.1.1960 bei einem Autounfall ums Leben kam, im Bewußtsein [sic] seiner Zeitgenossen - die wir alle sind - zu verblassen beginnen. ${ }^{1}{ }^{1}$ Bartsch's Beitrag, auf den noch eingegangen wird, konnte die Verdrängung von Camus' politischem Vermächtnis nicht aufhalten. Die Ursachen für diese zunehmende Verdrängung sind umfassend analysiert ${ }^{2}$ und liegen zweifellos darin begründet, dass führende linke Intellektuelle - allen voran Jean-Paul Sartre - an Camus' politischphilosophischem Hauptwerk Der Mensch in der Revolte ${ }^{3}$ vernichtende Kritik geübt hatten: Dieses wäre - so die Vorwürfe kurz zusammengefasst - unsystematisch, unphilosophisch und überdies einer dubiosen, unrealistischen Moral geschuldet, welche gesellschaftliche Veränderungen verunmögliche. ${ }^{4}$ Mit der Ausgrenzung aus dem Kreise der sozialistischen Denker verlor sich aber auch das Interesse an Camus' politischem Werk insgesamt. Denn wer sonst hätte sich mit dem Philosophen des Absurden und der Revolte beschäftigen sollen, der von sich selbst behauptete: »Ich gehöre zur Linken: mir und ihr zum Trotz «? ${ }^{5}$ So wurde Camus in der literarischen Rezeption zwar großes Interesse und geradezu Begeisterung zuteil, sein politisches Denken allerdings fand - von wenigen Ausnahmen ${ }^{6}$ abgesehen - über viele Jahrzehnte hinweg kaum Beachtung. Erst in den letzten Jahren ${ }^{7}$ und insbesondere aus Anlass seines 100. Geburtstags am 7. November 2013 haben sich einige wenige

1 Bartsch 1965, S. 787.

2 Vgl. Aronson 2005.

3 Vgl. ebd.

4 Vgl. Todd 1999, S. 605 f.

5 Camus 1989, S. 27.

6 Vgl. Guérin 2013.

7 Vgl. Hayden 2013; Sändig 2004.

Leviathan, 42. Jg., 2/2014, S. 249- 266 
etwas eingehender damit beschäftigt. ${ }^{8}$ Camus' Relevanz und Aktualität für die politische Theorie und die Politikwissenschaft hat etwas zu bieten, woraus man die Grundzüge einer Demokratietheorie der Revolte entwickeln kann.

\section{Politische Aspekte im Werk von Albert Camus}

Um die demokratietheoretischen Anknüpfungspunkte ${ }^{9}$ bei Camus zu veranschaulichen, muss man einige zentrale Gedanken aus seinem umfangreichen Werk herausdestillieren.

\subsection{Die existenzielle Absurdität}

Die erste Grundannahme Albert Camus' lautet, dass der Mensch in eine sinnlose Welt hineingeboren wird - eine Ansicht, die er mit Vertretern der Existenzialphilosophie teilt. ${ }^{10}$ Die Annahme ist simpel, aber folgenreich: Es gibt keinen objektiven Sinn in unserem Dasein. Die Welt ist absurd. Es gibt weder einen Beweis für Gott noch einen Grund, »sich das Ende der Geschichte auszudenken «. ${ }^{11}$ Wer nun aber nicht an Gott und nicht an Ideologien glaubt, ist auf sich selbst zurückgeworfen. Es bleiben weder objektive Ziele der Schöpfung noch solche der Menschheit. ${ }^{12}$ In Anbetracht dieser Sinnlosigkeit stellt sich für Camus eine zentrale Problematik, nämlich jene des Selbstmords. Die einzige Frage von philosophischer Relevanz sei jene, warum man in einer absurden Welt überhaupt weiterleben sollte. ${ }^{13}$ Dieser Frage geht er in seinen beiden philosophischen Hauptwerken Der Mythos des Sisyphos (1942) und Der Mensch in der Revolte (1951) nach. Seine Antwort ist klar und doch paradox, denn sie ist lebensbejahend. Man dürfe sich nicht umbringen,

8 Die 2012 in Frankreich und 2013 auf Deutsch erschienene Biographie von Michel Onfray L'ordre libertaire. La vie philosopique d'Albert Camus (auf Deutsch erschienen unter dem Titel Im Namen der Freiheit. Leben und Philosophie des Albert Camus) stellt die politischen Aspekte im Werk und Leben des französischen Nobelpreisträgers in den Mittelpunkt und zeichnet ihn als libertären Anarchisten; vgl. Onfray 2013. Diese anarchistische Ausrichtung wurde zuvor auch von anderen Autoren betont, beispielsweise Marin 1998; Guérin 2009. Auch der Schweizer Philosoph und Publizist Martin Meyer (2013) hat sich aktuell mit dem Leben und Werk Camus' beschäftigt.

9 Die Frage, wann eine Theorie als demokratie- oder politikrelevant betrachtet werden kann, ist in der Politikwissenschaft nicht eindeutig geklärt; vgl. Brodozc, Schaal 2001; Thiery 2009; Hartmann, Offe 2011.

10 Camus sah sich selbst allerdings nicht als Existenzialisten. Er kritisierte an Heidegger die kühle und pessimistische Haltung und versuchte einen optimistischeren Gegenentwurf; vgl. Camus 1959; Todd 1999.

11 Camus 1997 [1953], S. 254.

12 Ebd., S. 35.

13 Camus 1959 [1956], S. 9. 
weil man mit dem Selbstmord der Absurdität Recht geben würde. Nur ein Mensch, der am Leben sei, könne behaupten, die Welt sei absurd. ${ }^{14}$

»Das Absurde in sich selbst ist Widerspruch. Es ist ein Widerspruch seinem Inhalt nach, denn es schließt die Werturteile aus und will dennoch das Leben aufrechterhalten, wo doch Leben an sich schon ein Werturteil ist. [...] Jede Philosophie der Nicht-Bedeutung ruht auf dem Widerspruch gerade der Tatsache, die sie ausspricht. Die einzige widerspruchslose Haltung, gründend auf der Bedeutungsleugnung, wäre das Schweigen, wenn es nicht seinerseits etwas bedeutete. ${ }^{15}$

Es geht ihm also um die Frage des Weiterlebens in einer sinnlosen Welt. Weiterzuleben heiße, gegen deren Absurdität zu revoltieren im vollen Bewusstsein, dass die Revolte nie ganz gelingen kann, und im vollen Bewusstsein des unauflösbaren Widerspruchs: Ich revoltiere, also bin ich beziehungsweise »Ich empöre mich, also sind wir «. ${ }^{16}$ Im Mythos des Sisyphos beschreibt Camus, wie dieser als Strafe der Götter einen Stein stets auf den Berg rollen muss und dabei weiß, dass er es nie ganz schaffen, der Stein immer wieder hinunterrollen wird. Dies spiegle die menschliche Existenz wider. Indem Sisyphos die Strafe annimmt und den Stein immer aufs Neue nach oben schiebt, revoltierte er gegen die absurde Strafe der Götter. Würde er sich töten, hätte die Absurdität gesiegt. Man müsse sich Sisyphos als einen glücklichen Menschen vorstellen. ${ }^{17}$

"[...] Camus entlarvt den Selbstmord als ebenso absurd wie die Absurdität, die aufzulösen er vorgibt. Mit der gegen sich selbst gerichteten Tat glaubt man, die Absurdität des Lebens aufzuheben, während man sie paradoxerweise erst bekräftigt und ihre Macht noch vergrößert. Das Leben ist absurd, genauso absurd aber ist, es zu beenden. Was also bleibt? Leben. $« 18$

Von dieser existenziellen Annahme eines absurden Lebens aus entwickelt Camus seine Gedanken über die sinnlose Existenz, derer sich das Individuum bewusst werde, und über die Frage, wie mit dieser sinnlosen Existenz umzugehen sei und was sie für die Gesellschaft und die Politik bedeute.

\subsection{Revolte gegen Ungerechtigkeit und Unfreiheit}

Die Revolte des Einzelnen gegen die Absurdität der Welt sei das Weiterleben, aber sie bliebe nicht darauf beschränkt. »Ich rufe, daß ich an nichts glaube und daß alles absurd ist, aber ich kann an meinem Ausruf nicht zweifeln, und zum mindesten muß ich an meinen Protest glauben. ${ }^{19}$ Der erste Akt der Revolte sei also das Leben selbst als Antwort auf die Sinnlosigkeit, der man als Individuum ausgesetzt ist. Die Revolte, so Camus, bejaht das Leben und die Freiheit und lehnt ab, was diese beiden bedroht. Er führt das Beispiel des Sklaven an, der eines Tages »Nein« sagt, gegen

14 Camus 1997 [1953], S. 15.

15 Man fühlt sich bei dieser Passage an das konstruktivistische Diktum »Man kann nicht nicht kommunizieren « erinnert (Watzlawick 2011).

16 Camus 1997 [1953], S 31.

17 Camus 1959 [1956], S. $98 \mathrm{f}$.

18 Onfray 2013, S. 226.

19 Camus 1997 [1953], S. 17.

Leviathan, 42. Jg., 2/2014 
den Herrn aufbegehrt und eine Grenze zieht. Er sagt Nein zu den Erniedrigungen und zur Unterdrückung und damit gleichzeitig Ja zu einem Teil seines Selbst. »Wer revoltiert, im etymologischen Wortsinn, macht kehrt. Er schritt unter der Peitsche des Herrn. Nun bietet er ihm die Stirn. «20 Der Widerstand und das Aufbegehren begännen zuerst im Einzelnen. Aber die Revolte gehe darüber hinaus. Denn in der Tatsache, dass alle Menschen der Absurdität gleichermaßen und damit als Gleiche ausgeliefert seien, begründe sich eine universelle Solidarität unter revoltierenden Leidensgenossen der Spezies Mensch. ${ }^{21}$ Da jedes menschliche Wesen mit der existenziellen Absurdität konfrontiert sei, könne die Revolte, die den eigenen Tod ablehnt, auch den Tod anderer nicht akzeptieren. Revoltieren sei somit zugleich ein Akt der individuellen Befreiung, des individuellen Widerstands wie auch einer der Solidarität unter Gleichen. Die Ablehnung des Selbstmords beinhaltet für Camus damit schon die Ablehnung des Mordes. Daraus leitet sich für ihn die Revolte im Hier und Jetzt gegen jede Form der Ungerechtigkeit und der Unterdrückung ab, wird aber nicht zur Forderung nach vollständiger, absoluter Freiheit. ${ }^{22}$

"Weit entfernt, eine allgemeine Unabhängigkeit zu fordern, will die Revolte die Anerkennung der Tatsache, daß die Freiheit überall da eine Grenze habe, wo sich ein menschliches Wesen befindet, denn die Grenze ist eben die Macht der Revolte dieses Wesens. [...] Der Revoltierende verlangt ohne Zweifel eine gewisse Freiheit für sich selbst, aber keinesfalls, wenn er konsequent ist, das Recht, das Wesen und die Freiheit des andern zu vernichten. [...] Die Freiheit, die er fordert, fordert er für alle; diejenige, die er ablehnt, verbietet er allen. ${ }^{23}$

Freiheit und Gerechtigkeit müssten sich somit gegenseitig begrenzen. »Die absolute Freiheit ist das Recht des Stärkeren zu herrschen. Die absolute Gerechtigkeit schreitet über die Unterdrückung jedes Widerspruchs: sie zerstört die Freiheit. « ${ }^{24}$ In diesem Zitat steckt die von Camus immer wieder und zu verschiedenen Gelegenheiten geäußerte Kritik am Faschismus wie am Marxismus..$^{25}$ »Der erstere hat nie davon geträumt, den ganzen Menschen zu befreien, sondern nur davon, einige zu befreien

20 Ebd., S. 22.

21 Ebd., S. 30.

22 Ebd., S. 320.

23 Ebd., S. $320 \mathrm{f}$.

24 Ebd., S. 324.

25 Während seine Kritik am Faschismus und Nationalsozialismus sowie sein Kampf im Widerstand gegen Hitler ihm unter allen Intellektuellen stets zur Ehre gereicht haben, wurde ihm seine Kritik am Stalinismus in der französischen Linken massiv angekreidet. Der erste Vorwurf erfolgte in einer Besprechung des Buchs Der Mensch in der Revolte in den von Jean-Paul Sartre herausgegebenen »Les Temps modernes « durch Francis Jeanson. Dieser wirft Camus vor, eine metaphysische Abhandlung gegen die Revolution geschrieben und damit jede berechtigte Forderung nach Umsturz und Veränderung ungerechter Zustände geleugnet zu haben. Camus glaube nicht an die Macht der Infrastruktur und der Herrschaftsverhältnisse (Todd 1998, S. 605 f.; Aronson 2005, S. 231 f.). Er wende sich gegen die Revolution, obwohl diese oft die einzige Hoffnung der Menschen sei (ebd.). Diese Kritik, die den Bruch zwischen Camus und Sartre begründete, hielt sich hartnäckig und wurde in den folgenden Jahrzehnten oft wiederholt. Camus wehrte sich 
durch die Unterjochung der andern. Der letztere strebt in seinem tiefsten Prinzip danach, alle Menschen zu befreien, indem er sie alle vorübergehend knechtet. «26

Die Revolte richtet sich bei Camus gegen alle totalitären Herrschaftssysteme. Sie verliere aber dort ihre Legitimität, wo sie ihre eigenen Prinzipien bricht - und dies geschieht an jenem Punkt, wo sie zu den Waffen greift. Camus stellt hier die Revolution der Revolte gegenüber. An den historischen Beispielen der Französischen und der Russischen Revolution argumentiert er, dass die Revolution mörderisch sei und damit die Prinzipien der Revolte missachte: »Die meisten Revolutionen gewinnen ihre Form und Originalität durch einen Mord. Alle, oder fast alle, sind menschenmörderisch «. ${ }^{27}$ Camus, der sich selbst im Widerstand engagierte, der sich freiwillig zum Militärdienst gegen Hitler gemeldet hatte, aber aufgrund seiner Tuberkulose untauglich war, lehnte Gewalt als Mittel zur Selbstverteidigung nicht grundsätzlich ab. Er wandte sich aber gegen die geplante Tötung von Menschen aus Prinzip. Und diese geplante und brutale Tötung warf er den Jakobinern wie den russischen Revolutionären vor. Sie verstoße gegen die existenziell begründeten Prinzipien der Revolte. ${ }^{28}$

Diese Revolte richte sich zum einen gegen die Unfreiheit, wie im Beispiel des neinsagenden Sklaven deutlich wird. Sie richte sich aber auch gegen Ungerechtigkeit. Camus meint damit nicht nur die Ungerechtigkeiten in Hinblick auf politische Rechte, er bezieht die sozialen und ökonomischen Ungerechtigkeiten mit ein. In Der Mensch in der Revolte stellt er Karl Marx den Marxisten gegenüber und lobt Marx' Fähigkeit, sich in die Arbeiter hineinzuversetzen, ${ }^{29}$ auch wenn er dessen Idee der

in einer Replik mit dem Hinweis darauf, dass er stets die historischen und ökonomischen Ungerechtigkeiten angeprangert und bekämpft habe, und wirft dem Rezensenten vor, ihn bewusst missverstanden zu haben (Aronson 2005, S. 234). Und tatsächlich scheint Jeansons Rezension die Thesen Camus' völlig zu verdrehen. Denn die Prinzipien der Revolte bedeuten bei Camus keineswegs, wie ihm vorgeworfen wird, die Leugnung oder das Akzeptieren ungerechter Verhältnisse. Sie bedeuten die Ablehnung des Mordes bei gleichzeitiger Auflehnung und Widerstand gegen Unfreiheit und Ungerechtigkeit. Wenn Camus von » Revolution « spricht, dann meint er die historischen Beispiele, an denen er sich abarbeitet, nämlich die Französische und die Russische Revolution.

26 Camus 1997 [1953], S. 277.

27 Ebd., S. 128.

28 Camus betont, dass die Revolte gegen Unterdrückung und gegen die Brutalität der Obrigkeit verständlicherweise rasch an einen Punkt kommen könne, wo sich die Frage nach der Anwendung von Gewalt stellt. Am Beispiel der russischen Sozialrebellen Ende des 19. und Anfang des 20. Jahrhunderts macht er deutlich, wie zerrissen der Revoltierende in Anbetracht einer solchen Situation sein kann. Während kühle Revolutionäre der unbestimmten Gewalt ohne Zaudern freien Lauf ließen, reiße es andere zwischen der gewaltlosen Revolte und der gewalttätigen Revolution wenigstens noch hin und her. Der russische Student Kaliayew etwa weigerte sich, die Bombe auf den Großfürsten zu werfen, weil dessen Kinder und andere Unschuldige hätten verletzt oder getötet werden können. Camus verteidigt diese Terroristen nicht, aber er verweist auf den Unterschied zwischen diesen » zartfühlenden Mördern « und den berechnend kaltblütigen (ebd., S. 194 f.).

29 Ebd., S. 236.

Leviathan, 42. Jg., 2/2014 
klassenlosen Gesellschaft als historisches Ziel verwirft. Simone Weil stimmt er darin zu, dass die Stellung des Arbeiters doppelt unmenschlich sei, weil sie Geld und Würde entbehre. ${ }^{30}$ Ein deutliches Bild von Camus' Vorstellungen einer gerechten Gesellschaft ergibt sich neben diesen Passagen aus seinen journalistischen Artikeln, seinen Reden und seinen Tagebuchaufzeichnungen. Seine Forderungen beinhalteten unter anderem die Abschaffung der Lohnarbeit und die Verstaatlichung wichtiger Wirtschaftszweige. Er sympathisierte mit dem Anarcho-Syndikalismus.

»Wir dürfen die Reformen nicht mit Blick auf eine noch weit entfernte Gesellschaft geringschätzen, dürfen aber trotz der Reformen auch das letzte Ziel nicht aus den Augen verlieren, das in der Einsetzung der Arbeiterklasse in all ihre Rechte durch die Abschaffung der Lohnarbeit besteht. Früher oder später muss der Widerstand der Privilegierten dem Allgemeininteresse weichen. Das wird eher früher als später der Fall sein, wenn wir ab sofort damit beginnen, die Gewerkschaften an der Verwaltung des Volkseinkommens zu beteiligen. « ${ }^{31}$

Die Auflehnung gegen die Absurdität der Welt beinhalte somit die Revolte gegen Unfreiheit und Ungerechtigkeit. Im Gegensatz zur Revolution bleibe sie aber auf die Gegenwart bezogen. Kein Heilsversprechen für die Zukunft könne eine Tötung in der Gegenwart legitimieren. Damit erteilt Camus nicht nur den religiösen Vorstellungen eines Lebens nach dem Leben, sondern auch den absoluten politischen Utopien eine Absage.

\subsection{Zweifel und Dialog}

Aus der Absurdität und der Revolte ergeben sich zwei weitere Aspekte, die im Werk von Albert Camus immer wieder auftauchen und politisch höchst relevant sind. Dies sind zum einen der Zweifel und zum anderen der Dialog. Der Zweifel an fixen und unabänderlichen Wahrheiten gehört zu den Grundannahmen bei Camus. Nur wer an den jenseitigen und diesseitigen Wahrheiten und Heilsversprechungen zweifle, könne gegen sie revoltieren. Zu dieser Haltung gehöre aber auch der Selbstzweifel. In einem Vortrag aus dem Jahre 1948, den Camus auf Einladung eines Dominikanerklosters hielt, wird dies deutlich. Er weist darauf hin, dass er sich »nicht im Besitz irgendeiner absoluten Wahrheit oder einer Botschaft « fühle. ${ }^{32}$ Dies entspricht dem, was er wenige Jahre später in Der Mensch in der Revolte wiederholt. Der Zweifel, die Zerrissenheit, die Unsicherheit gegenüber der Sinnfrage stehen am Beginn. ${ }^{33}$ Nur wer zweifle, verwerfe endgültige Lösungen jeglicher Art. Camus verwarf nicht nur die faschistischen und marxistischen Versprechungen, er sprach sich auch wiederholt und konsequent gegen die Todesstrafe aus. Denn auch sie gründet auf Gewissheiten, die er bezweifelte. ${ }^{34}$ In seinen Betrachtungen zur Todesstrafe kommt seine Haltung einprägsam zum Ausdruck: »Kann man uns mit Bestimmtheit sagen, daß

30 Ebd., S. 245.

31 Camus, zitiert nach Onfray 2013, S. 400.

32 Camus 1997 [1960], S. 66.

33 Camus 1997 [1953], S. 17.

34 Ebd., S. 326. 
keiner der Hingerichteten für die Gesellschaft zu retten gewesen wäre? «35 Nein, das könne man nicht, da es keine Gewissheit gebe. Die Todesstrafe bezeichnet Camus unter anderem deswegen als Skandal, weil sie jeden Zweifel ausschließe und jede Wiedergutmachung verunmögliche. Sie sei absolut und nicht rückgängig zu machen. »Einen Menschen zum Verlust seines Lebens verurteilen, heißt so viel wie bestimmen, daß dieser Mensch keinerlei Aussicht mehr hat, wiedergutzumachen. «36 Wer wie Camus an absoluten Wahrheiten zweifelt, müsse die Todesstrafe ablehnen, und zwar aus demselben Grund, wie er Revolutionen ablehnt.

Wer absolute Wahrheiten bezweifelt, bezweifelt gleichzeitig auch den Monolog. Daher spricht sich Camus an vielen Stellen seines Werks direkt oder indirekt für den Dialog aus. So auch in dem schon zitierten Vortrag bei den Dominikanern. »Vielmehr möchte ich Ihnen heute sagen, daß die Welt ein echtes Zwiegespräch nötig hat, daß das Gegenteil eines Dialogs ebenso gut Lüge heißt wie Schweigen und daß ein Zwiegespräch deshalb nur zwischen Menschen möglich ist, die das bleiben, was sie sind, und die wahr sprechen. «37 Den Standpunkt anderer anzuhören und mit einzubeziehen ${ }^{38}$ ist daher die konsequente Folge aus allem bereits Genannten: der Absurdität, der Revolte und des Zweifels. Unterstrichen wird die Betonung des kritischen Dialogs auch durch seine Aussagen zur Rolle des Journalismus. Kritik und Selbstkritik seien dessen Aufgaben sowie die Hervorbringung einer kritischen Öffentlichkeit. ${ }^{39}$ "Auf der Bühne wie im Leben geht der Monolog dem Tod voraus. « ${ }^{40}$ In einem frühen Stück über den römischen Kaiser Caligula lässt Camus seine Hauptfigur über weite Strecken monologisieren, denn der Monolog ist die Methode der undemokratischen Herrschaft. Um jeden Zweifel am Gesagten von vornherein im Keim zu ersticken, lässt der Diktator niemanden als sich selbst zu Wort kommen. Die Methode der Revolte hingegen sei der Dialog, der Widerspruch. Camus liefert mit seinem Werk einen existenzialphilosophischen Beitrag zu einer Diskursethik, die das Schweigen der Welt gegenüber dem menschlichen Fragen beklagt und daraus die Notwendigkeit des Dialogs ableitet. ${ }^{41}$ Er weist auf grundlegende Gegensätze hin: Revolte vs. Revolution, Zweifel vs. absolute Wahrheit und Dialog vs. Monolog.

\subsection{Solitaire und solidaire: Einsam, fremd und solidarisch in einer globalen Demokratie}

Ein weiterer Gedanke in Camus' Werk, der für sein politisches Denken relevant ist, ist die Fremdheit. Der Mensch, der nicht an Gott und nicht an ein endgültiges Ziel

35 Camus 1997 [1960], S. 135 f.

36 Ebd., S. 144.

37 Ebd., S. 66.

38 Isaac 1992, S. 170.

39 Camus nach Onfray 2013, S. 292;

40 Camus 1997 a [1953], S. 320.

41 Vgl. dazu Hartmut Rosa in der Frankfurter Rundschau vom 18. Juni 2011. www.fronline.de/kultur/zeitdiagnose-ist-da-draussen-jemand-,1472786,8569942.html (Zugriff vom 25.11.2013). 
der Geschichte glaubt, der auf sein Fragen nach dem Sinn dieser Welt keine Antwort bekommt, sei ein Fremder. Dieser Fremde sei das Sinnbild der absurden Existenz. Meursault, die Hauptfigur im Roman Der Fremde, steht für eine unangepasste Person, die sich nicht normkonform verhält, ${ }^{42}$ sondern nur in den Tag hinein lebt und alles hinnimmt, wie es kommt. Allerdings fehlt ihm ein wesentlicher Aspekt, um ein Mensch in der Revolte zu sein: Er begehrt nicht gegen Ungerechtigkeit und Unfreiheit auf, sondern begeht im Gegenteil ganz indifferent einen Mord. Er ist einsam und fremd, ein solitaire. Ihm fehlt aber das, was Camus fast zehn Jahre später in Der Mensch in der Revolte als wesentliche Eigenschaft des Revoltierenden bezeichnet und was er in seinem weiteren großen Roman Die Pest literarisch verarbeitet, nämlich die Solidarität. Solitaire und solidaire - das Individuum sei einsam und fremd in einer absurden Welt, eine Erfahrung, die zu Revolte und zu Solidarität führe. Man müsse sich nicht schämen, das Glück der Solidarität vorzuziehen, sagt die Hauptfigur Dr. Rieux zum Journalisten Rambert in Die Pest, woraufhin dieser antwortet, dass man sich aber dafür schämen könne, alleine glücklich zu sein. ${ }^{43}$ Solange andere leiden, unterdrückt und ungerecht behandelt werden, kann Camus' Revoltierender nicht ruhen. Die Revolte bejaht nicht nur das eigene Leben, sondern das Leben aller, und daraus entsteht Solidarität. Diese Solidarität geht bei Camus über alle Grenzen hinweg, über nationalstaatliche wie über religiöse oder weltanschauliche. Der Mensch in der Revolte sei gleichzeitig einsam, fremd und solidarisch. Die Konsequenz daraus ist die Forderung nach weltweiter Gerechtigkeit und gleicher Freiheit für alle.

Der dazu passende Gedanke einer europäischen Föderation und einer darauf folgenden internationalen Demokratie kommt insbesondere in Camus' kürzeren Texten und seinem journalistischen Engagement zum Ausdruck. Die europäische Einigung lag ihm schon während der Kriegsjahre besonders am Herzen. Er bezeichnet Europa in seinem »Brief an einen deutschen Freund « als die große Hoffnung, die ihn und seine Kameraden im Kampf gegen Hitler aufrecht hielt. ${ }^{44}$ Ein Europa, das nach dem Kriege geschaffen werden müsse. In den 1950er Jahren bringt er diese Idee erneut zum Ausdruck und konkretisiert sie. Er beschreibt seine Identität als Algerier, Franzose und Europäer. »Ich habe [...] mit der Selbstverständlichkeit, mit der man atmen lernt, die Erfahrung gemacht, daß die Liebe zur engeren Heimat sich weiten kann, ohne zugrunde zu gehen. Und schließlich fühle ich mich als Europäer, weil ich mein Land liebe. « 45 »Einheit und Vielfalt « sei die Formel Europas. Camus schwebte eine europäische Föderation vor, der auch Russland angehören würde. ${ }^{46}$ Dieses Europa sollte ein Europa der Revolte sein und in eine globale, universale Demokratie münden. Universalität bekommt dabei eine doppelte Bedeutung. Sie bezieht sich zum einen auf die universell gültige Problematik der menschlichen Exis-

42 Vgl. Camus 1957.

43 Vgl. Camus 1998 [1948].

44 Camus 1997 [1960], S. 23.

45 Ebd., S. 219.

46 Ebd., S. 220. 
tenz und zum anderen auf eine globale und damit universelle Vernetzung in Hinblick auf politische Interdependenzen und Abhängigkeitsverhältnisse. In einem Artikel aus dem November 1948 mit dem Titel »Démocratie et dictature internationale « heißt es: "Das Brot Europas stammt aus Buenos Aires, die Arbeitsmaschinen in Sibirien stammen aus Detroit. Heute ist die Tragödie kollektiv. [...] Wir wissen daher ohne den Schatten eines Zweifels, dass die neue Ordnung, die wir suchen, nicht nur national oder kontinental, noch viel weniger westlich oder östlich sein kann. Sie muss universell sein $"{ }^{47}$ Camus spannt hier den Bogen zwischen seinem politik-theoretischen Werk und seinen konkreten Forderungen als Journalist und Intellektueller. Er schließt in Bezugnahme auf die Vereinten Nationen, dass es sich bei dieser Organisation um eine internationale Diktatur handle, da das internationale Gesetz beziehungsweise das Völkerrecht nicht wie in Demokratien üblich von der Legislative, sondern von der Exekutive gemacht werde. Um die internationale Diktatur zu beenden, forderte er weltweite Wahlen und ein Weltparlament. »[...] so lange wir dieses Parlament nicht haben, müssen wir uns gegen die internationale Diktatur auflehnen, und zwar mit internationalen Maßnahmen, die dem verfolgten Ziel nicht widersprechen. «48

\section{Demokratietheoretische Konsequenzen aus dem Werk Albert Camus'}

Die zentralen Gedanken in Albert Camus' Werk lassen sich für die politische Theorie und insbesondere für die Demokratietheorie fruchtbar machen. Entlang der oben genannten Aspekte ergibt sich aus seinen Schriften eine für die Demokratietheorie interessante Argumentationskette, die, von der einsamen Absurditätserfahrung des Individuums ausgehend, bei den existenziellen Bedingungen des Menschseins beginnt und bei der Demokratie als »bester « Staatsform endet.

\subsection{Demokratie als Staatsform des Absurden und der Unabschließbarkeit}

Die erste Annahme, nämlich jene einer absurden Welt, in der es kein finales Ziel gibt, ist bereits im demokratischen Gedanken grundsätzlich angelegt, aber in keiner der gängigen Demokratietheorien ausgesprochen. Die Entstehungsgeschichte der modernen Demokratie jedoch ist die Geschichte der Revolte gegen einen von Gott legitimierten Herrschaftsapparat. Das Gottesgnadentum wird im Zeitalter der Aufklärung infrage gestellt und in der Französischen Revolution schließlich offen bekämpft. Diese gedankliche Entwicklung war möglich, weil Humanismus und Renaissance den Weg zum Zweifel bereitet haben, der auch den Zweifel an der Göttlichkeit, jedenfalls aber der göttlichen Legitimation der Herrscher und schließlich die Entstehung einer kritischen Öffentlichkeit beinhaltete. ${ }^{49}$ Dieser Zweifel wird paradoxerweise Grundlage einer Überzeugung; nämlich jener, dass keine Weltanschauung, keine Jenseitsvorstellung und kein Heilsversprechen objektive Richtigkeit

47 Camus 1950, S. 160; Übersetzung M.P.

48 Ebd., S. 166; Übersetzung M.P.

49 Vgl. Habermas 1968.

Leviathan, 42. Jg., 2/2014 
beanspruchen können. Er ist damit historische Grundlage für die Erkenntnis der Absurdität der Welt. Um diese anzuerkennen, muss man kein Atheist sein. Es reicht aus, was der italienische Müller Menocchio ${ }^{50}$ schon im 16. Jahrhundert zum Ausdruck brachte: Wir können nicht mit Gewissheit sagen, was wahr und richtig ist. Daher solle sich die weltliche Macht gegenüber den transzendenten Vorstellungen neutral verhalten. Dieser Gedanke wird später zu einer weitgehend anerkannten Prämisse der modernen politischen Theorie und findet durch die Trennung von Kirche und Staat seinen Ausdruck, ${ }^{51}$ am prägnantesten in der Laizität der französischen oder türkischen Verfassung.

Die Neutralität des modernen Staates gegenüber Religionen und Weltanschauungen ist vom Gedanken der Absurdität nicht weit entfernt. Auch wenn in keiner Verfassung von der Sinnlosigkeit der Welt die Rede ist und sich manche sogar ausdrücklich auf eine Religion beziehen, so steckt in vielen von ihnen doch die Grundannahme, die lautet: Der Sinn der Welt lässt sich nicht objektiv erschließen. Diese Annahme ist die Voraussetzung für die Existenz demokratischer Systeme in unserem heutigen Verständnis.

Mit der radikalen Anerkennung der Absurdität unserer Welt, wie sie bei Camus geäußert wird, und seiner Leugnung einer geschichtlichen Logik oder Sinnhaftigkeit, die uns zu einem finalen Ziel führen könnte, ist die Konzentration des Lebens und der Politik auf das Hier und Jetzt folgerichtig. Die Demokratie ist die Staatsform, die dieser Überzeugung Ausdruck verleiht. Nur eine Demokratie, die auf jegliches Heilsversprechen verzichtet und sich auf die Verteidigung von Freiheit und Gerechtigkeit in der Gegenwart bezieht, wird der existenziellen Absurdität und der ethischen Neutralität gerecht. Die Diskrepanz zwischen verfassungsrechtlichen Grundsätzen und Realpolitik ist Camus dabei freilich nicht entgangen. Darum betonte er in einem journalistischen Artikel: "Die Demokratie ist nicht die Herrschaft der Mehrheit, sondern die Beschützung der Minderheiten ".52 Er unterstreicht damit, dass der Staat dort die Demokratie verletze, wo er der Sinndefinition einer Mehrheits-Gruppe größeren Wert einräumt als jener der Minderheit. ${ }^{53}$ Die Beschützung der Möglichkeit anderer Sinndefinitionen ist demnach grundlegende Aufgabe der Demokratie und die Annahme einer objektiv nicht herstellbaren Sinnhaftigkeit der Welt, ihrer Absurdität also, ihre Voraussetzung. Anders formuliert: Nur weil die Welt absurd ist, brauchen wir eine Demokratie. Was daraus folgt, ist die Ablehnung von ideologischen Zielen, also die Unabschließbarkeit des Politischen.

\subsection{Demokratie als Staatsform der Revolte für Freiheit und Gerechtigkeit}

Aus der Absurdität folgt die Revolte. Dieser Schritt lässt sich nicht unpolitisch deuten. Was aber heißt die Revolte für die politische Theorie? Wenn die Demokratie, wie oben argumentiert, die Staatsform ist, die als einzige der Absurdität der Welt

50 Vgl. Ginzburg 1979.

51 Vgl. Lipset, Rokkan 1967.

52 Camus 1989, S. 260.

53 Siehe auch Rey 2006, S. 272 f. 
gerecht wird, dann muss sie zwangsweise die Revolte zulassen, ja muss sie fördern, denn diese gehört zur Absurdität dazu. Demokratie beruht auf zwei Prinzipien: Freiheit und Gerechtigkeit. ${ }^{54}$ Die Revolte ist die Methode zur Erreichung und Verteidigung dieser Prinzipien.

Die existenzialistische Grundannahme Camus' lässt sich anhand eines Bildes darstellen, und zwar mithilfe einer Umdeutung der Heidegger'schen Annahme des »Indie-Welt-Geworfen-Seins «. Während Heidegger von einem in Bezug auf das Kind unfreiwilligen, passiven Geburtsakt ausgeht - das Kind wird "geworfen « -, kann die Camus'sche Idee der Revolte schon am Anfang jeder Existenz das Aktive hervorkehren. Die Geburt ist dann die erste Revolte, die zur Freiheit und zum Leben führt. Das Ungeborene lässt sich nicht passiv »werfen «, sondern revoltiert gegen die Enge des Mutterleibs und bricht in die Freiheit auf. Bliebe es passiv, so würde es sterben. Diese Erfahrung steht am Beginn jedes Menschenlebens. Der Mutterleib gibt dem Freiheitsdrang ihrer Leibesfrucht nach und erlaubt den Widerstand. Das Leben beginnt so mit einer Revolte, die zur Freiheit führt: »Ich revoltiere, also bin ich. « Die existenzielle Erfahrung der erfolgreichen Revolte eine alle Menschen und begründe deren Solidarität. Symbolisch gesprochen, wiederholt sich dieser Prozess immer dann, wenn jemand gegen einen äußeren Zwang aufbegehrt. Die endgültige Freiheit ist im absurden Leben unerreichbar, wie Sisyphos weiß. Politisch gesprochen, stellt sich die Frage, ob ein Staat als Zwangsapparat die Revolte zulässt oder im Keim erstickt und damit, symbolisch gesprochen, jedes freie Leben tötet. Revolte, Leben und Freiheit gehören aus dieser Perspektive zusammen. Und die Demokratie ist, um in diesem Bild zu bleiben, der Mutterleib, der die Revolte zulässt.

Gleichzeitig leitet sich aus der Revolte gegen Unfreiheit und Unterdrückung auch die Forderung nach Gerechtigkeit ab, denn gleiche Freiheit erfordert die gerechte Verteilung von Lebenschancen und Ressourcen. Für Camus sind die wirtschaftlichen Verhältnisse nicht von den politischen abzukoppeln. Hier trifft er sich mit den meisten sozialistischen Theorien. Sein Argument für gerechte Verteilung gründet aber, wie beschrieben, in der Forderung der existenziellen Revolte. Nein sagen und revoltieren zu können muss allen Menschen gleichermaßen möglich gemacht werden. Aus diesem Grunde präferierte Camus einen anarcho-syndikalistischen Zugang, wie er bei Pierre-Joseph Proudhon ${ }^{55}$ oder Michail Bakunin angelegt war, der gleiche Machtverteilung und institutionelle Enthierarchisierung beinhaltet.

»Die Freiheit wählen, das heißt nicht, gegen die Gerechtigkeit wählen, wie man uns dies weismacht. Im Gegenteil, man wählt die Freiheit heute nur auf der Ebene derer, die allenthalben leiden und kämpfen, dort und nur dort. Man wählt sie zugleich mit der Gerechtigkeit, und wahrhaftig, in Zukunft ist es für uns nicht mehr möglich, die eine ohne die andere zu wählen. Wenn jemand euer Brot entzieht, beraubt er euch gleichzeitig eurer Freiheit. Aber wenn jemand euch eurer Freiheit beraubt, dann wißt, daß euer Brot bedroht ist, denn es hängt nicht mehr von euch und eurem Kampf ab, sondern von der Eigenmächtigkeit irgendeines Herrn. Je mehr die Freiheit in der Welt an Boden verliert, desto mehr wächst das Elend und umgekehrt. ${ }^{56}$

54 Vgl. Pelinka 2011.

55 Vgl. Proudhon 2010 [1840].

56 Camus 1997 [1960], S. 89. 
Camus spricht sich für eine gerechte Demokratie aus und impliziert, dass sich Sozialismus und Liberalismus gegenseitig bedingen - ein Gedanke, der vor ihm schon bei Jean Jaurès geäußert wurde. ${ }^{57}$

Von den derzeit gängigen Demokratietheorien passt Camus' Konzept am ehesten zur sozialen Demokratie, wie sie zuerst von Hermann Heller konzipiert wurde. ${ }^{58}$ Der Kritik an einer Demokratie, die keine Rücksicht auf soziale Fragen nimmt, hätte Camus wohl uneingeschränkt zugestimmt. »Er verteidigte die [damals gültige] Doktrin der Labour Party und den skandinavischen Sozialismus, weil ihm ein künftiges sozialistisches Europa eine gute Basis für das von ihm angestrebte föderale und libertäre Europa zu sein schien. «59

Wenn die Demokratie aber die Staatsform der Revolte im Sinne von Freiheit und Gerechtigkeit ist, so hat dies Folgen für ihre Institutionen und ihre Prozesse. Institutionen sind dann mit möglichst flachen Hierarchien auszustatten, die den Widerspruch, die Revolte, das Nein für jedes einzelne Individuum ermöglichen und zulassen. Effizienzargumente oder anderweitige Ziele müssten dem obersten Prinzip der Revolte untergeordnet werden. Das gilt für die UNO ebenso wie für Nationalstaaten und andere politische Einheiten, wobei letztere nach Camus föderale Subeinheiten einer weltweiten Demokratie sein sollten. Auch Interessenvertretungen und Unternehmen sind davon nicht auszunehmen. Als Vorbild gelten bei Camus demokratisch organisierte Gewerkschaften und die libertären Formen des Syndikalismus, die möglichst flache Hierarchien und gleiche Mitsprache für alle forderten. ${ }^{60}$ Dies bedeutet, dass es neben den Übereinstimmungen mit dem Konzept der sozialen Demokratie in Camus' Werk auch eine Nähe zu partizipatorischen Demokratiemodellen gibt, insbesondere zu John Dewey ${ }^{61}$ oder Carole Pateman ${ }^{62}$. Deren Idee einer weit über gewerkschaftliche Rechte hinausgehenden Workplace-Democracy, also einer Demokratisierung der Arbeitswelt bis in den Mikrokosmos der Unternehmen hinein, ist mit der Auffassung der Demokratie als Staatsform der Revolte gut vereinbar und trifft sich mit Camus' politischer Forderung nach flachen Hierarchien. Übereinstimmungen gibt es auch mit Hannah Arendts Idee der Demokratie als Lebensform. ${ }^{63}$

In Anbetracht einer zunehmenden Kritik an globalen Ungerechtigkeiten, eines Vertrauensverlusts in nationalstaatlich organisierte repräsentative Demokratien, eines Anstiegs an Protestbewegungen und einer lauter werdenden Globalisierungskritik ist die Revolte von besonderer Aktualität. Der Erfolg des Buchs Empört

57 Vgl. Jaurès 2012 [1898].

58 Vgl. Meyer 2005.

59 Onfray 2013, S. 400.

60 Ebd.

61 Vgl. Dewey 2008, der Demokratie nicht lediglich als Staats-, sondern als Lebensform begriff.

62 Vgl. Pateman 1970.

$63 \mathrm{Zu}$ den Ähnlichkeiten zwischen Arendt und Camus sowie deren Bezug zu Dewey vgl. Isaac 1992, S. 145 f. 
Euch! des französischen Intellektuellen Stéphane Hessel unterstreicht dies. Sein Aufruf, sich gegen Ungerechtigkeit und Unfreiheit im Hier und Jetzt aufzulehnen, bringt die Positionen und die Haltung Camus' sowie anderer französischer Philosophen der 1940er und 1950er Jahre in die aktuelle Debatten ein: »Das Grundmotiv der Résistance war die Empörung « ${ }^{64}$, führt er aus. Sein Essay endet mit den Worten: »Widerstand leisten, heißt Neues schaffen «. 65

Durch die Revolte wird, wenn man sie mit Camus als zentralen Aspekt einer Demokratie versteht, ein neuer Blick auf die kritische Zivilgesellschaft möglich. Sie entsteht dort, wo die einsamen Individuen zu solidarischen Kämpfern für eine gemeinsame Sache werden, wo die geteilte »solitude « in eine »solidarité « mündet, die jedenfalls für die Dauer des gemeinsamen Aufbegehrens anhält und sich bei jeder neuen Ungerechtigkeit wieder entfacht.

Widerstand und Protest der Rebellen, also das, was in der Politikwissenschaft häufig unter den Begriff der »unkonventionellen Partizipation « fällt, sind aus dieser Perspektive zentrale Indikatoren für die Qualität einer Demokratie und damit von besonderer Bedeutung. Isaac ist zuzustimmen, wenn er seine Analyse von Camus und Arendt mit der Aussage schließt, dass die Demokratie nicht nur Überzeugungen und Institutionen brauche, »sondern auch Rebellen des Geistes und der Vernunft, die couragiert genug sind, an Überzeugungen und Verbindlichkeiten zu rütteln und darauf zu bestehen, dass, wenn wir wirklich die menschliche Freiheit und Würde vorziehen, wir auch bereit sein müssen, mit unseren Unvollkommenheiten und Unterschieden zu leben $« .66$

\subsection{Demokratie als Staatsform des Zweifels und des Dialogs}

Die Demokratie ist die Staatsform, in der man Nein sagen kann, weil ihr der Zweifel immanent ist. Daraus folgt, dass sie wehrhaft gegenüber endgültigen Wahrheiten sein muss, um nicht in Gefahr zu geraten, einer diesseitigen oder jenseitigen Heilsversprechung geopfert zu werden. Camus unterstreicht, dass es zu den für einen Demokraten unverzichtbaren Tugenden zähle, dem Gegner zuzuhören. »Man müsste sich selbst ein wenig vergessen. Das würde den Abgeordneten und den Parteien ein wenig von dem verleihen, was die guten und wahren Demokratien ausmacht. Der Demokrat ist vor allem jemand, der zugibt, dass ein Gegner recht haben kann, der ihn daher sprechen lässt und der über seine Argumente nachzudenken bereit ist. Wenn die Parteien oder die Menschen so überzeugt von ihrer eigenen Vernunft sind, dass sie den Gegnern mit Gewalt den Mund stopfen, dann existiert die Demokratie nicht mehr. «67 Der Zweifel ist daher notwendig, auch ein Zweifel

64 Hessel 2011, S. 9.

65 Ebd., S. 21.

$66 »[\mathrm{~B}]$ ut also [...] rebels, of the mind and of the spirit, who are courageous enough to unsettle convictions and commitments and to insist that if we truly prefer human freedom and dignity, we must be willing to live with our imperfections and with our differences « (Isaac 1992, S. 259; Übersetzung M.P.).

67 Camus 1977, S. 320; Übersetzung M.P.

Leviathan, 42. Jg., 2/2014 
an sich selbst. Er ist an Fragen und Dialog gebunden und steht im Gegensatz zum Monolog. Camus formulierte dies mehrfach unter dem Schlagwort einer »bescheidenen Demokratie«: »Das demokratische Regime kann nur gefordert, geschaffen und bewahrt werden von Menschen, die wissen, dass sie nicht alles wissen. Der Demokrat ist bescheiden $[\ldots] \ll .68$

Daher ist die Demokratie als Staatsform der Revolte notwendigerweise auf dem Gespräch aufgebaut, auf dem Austausch von Argumenten und der Möglichkeit des Widerspruchs. Diese Annahme findet sich auch in einer Reihe von Demokratietheorien wieder, nicht nur in jenen, die sich mit politischen Diskursen und Fragen der demokratischen Kommunikation beschäftigen. Die Analyse hegemonialer und gegenhegemonialer Diskurse etwa, wie sie von Ernesto Laclau und Chantal Mouffe mit Rückgriff auf Gramsci unternommen wird ${ }^{69}$ deckt sich mit Camus' Forderung nach der Möglichkeit des Widerspruchs. Demokratien brauchen Widerspruch, müssen ihn zulassen. ${ }^{70}$ Sie benötigen den Dialog, den kommunikativen Austausch, die Deliberation, wie Habermas es betont. ${ }^{71}$ In funktionierenden Demokratien spielt der Widerspruch eine tragende Rolle und schlägt sich institutionell in der parlamentarischen Opposition nieder, außerinstitutionell in den sogenannten unkonventionellen Partizipationsformen wie Demonstrationen oder Streiks. ${ }^{72}$ Manipulative und populistisch angelegte Kommunikationsformen, die dem Monolog den Vorrang überlassen und Fragen oder Widerspruch nicht zulassen, sind in einer auf Camus basierenden Demokratiedefinition auszuschließen.

\subsection{Demokratie als Staatsform der Fremdheit und der internationalen Solidarität}

Da Camus stets vom existenziellen Leid des Individuums ausgeht, ist es konsequent, dass er nationale Grenzen als theoretisch irrelevante Größen ansieht, auch wenn er in seinen der Realität geschuldeten Analysen weiß, wie wirkmächtig diese sind. Dabei legt er ein von Staatszugehörigkeiten unabhängiges Gleichheitsprinzip an. Sein während der Algerienkrise geäußerter Vorschlag, allen Algeriern die französische Staatsbürgerschaft zu verleihen, ${ }^{73}$ entspricht seinen philosophischen Annahmen. Das Recht auf Revolte, auf Freiheit und Widerspruch gründet sich bei Camus nicht auf ein territorial begrenztes Gebiet eines Nationalstaates oder eine Staatsbürgerschaft, sondern auf die existenzielle Universalität des Menschseins. Da jeder Mensch vorerst fremd und einsam in eine absurde Welt tritt, gibt es keine Ausschließungsgründe vom grundlegenden Recht auf Revolte. Damit liefert Camus eine existenzphilosophische Begründung für ein universales Wahlrecht, jedenfalls aber für eine Verbesserung des Zugangs von MigrantInnen zu den politischen Rechten

68 Camus 1948; Übersetzung M.P.

69 Vgl. Laclau, Mouffe 1985.

70 Vgl. Bruell et al. 2009.

71 Vgl. Habermas 1992.

72 Vgl. Kaase 2000.

73 Onfray 2013, S. 472 f. 
ihres Aufenthaltslands, wie sie auch MigrationsforscherInnen fordern. ${ }^{74}$ Denn aus der existenziellen Leidenserfahrung heraus entsteht eine Solidarität, die sich in der Gleichheit politischer Rechte und damit in der Gleichheit auf das Recht der Revolte äußert. Ein Migrant, der in einem Land, in dem er sich aufhält, keine Möglichkeit hat, politische Rechte wahrzunehmen, ist seiner Stimme beraubt und kann nicht Nein sagen. Die internationale Solidarität geht aber darüber hinaus und besteht nicht nur gegenüber den politischen Rechten von MigrantInnen. Da es ohne Brot keine Freiheit gibt und ohne Freiheit das Brot gefährdet ist, ergibt sich bei Camus eine internationale Solidarität mit allen Menschen, die entweder das eine oder das andere entbehren. ${ }^{75}$ Die kapitalismuskritischen Bewegungen der Gegenwart, die sich für den Kampf gegen Armut und Hunger einsetzen, leiten ihre Motivation aus ähnlichen Erwägungen ab. Manche berufen sich sogar ausdrücklich auf Camus. ${ }^{76}$

\section{Konklusion: Die Konsequenzen einer Demokratie als Revolte}

Camus' Argumente legen nahe, dass die Demokratie jene Staatsform ist, die den existenziellen menschlichen Erfahrungen am ehesten entspricht: Versteht man die Geburt in einer Umdeutung von Heidegger nicht mehr als passives In-die-WeltGeworfen-Sein, sondern als erfolgreichen Akt der Revolte, der zu Leben und Freiheit führt, so folgt aus diesem existenzermöglichenden Erfolgserlebnis sowie der späteren, von Camus beschriebenen Absurditätserfahrung, dass die Revolte eine Lebensbedingung darstellt. Diese Lebens- und Freiheitsbedingung in eine Staatsform zu übersetzen bedeutet sodann, die Spielregeln beziehungsweise den Gesellschaftsvertrag daraufhin zu konzipieren. Die idealtypische moderne Demokratie trägt diesen Gedanken bereits in sich und kann als Staatsform der Revolte interpretiert werden, auch wenn sie in der Realität beständig an diesem Anspruch scheitert.

Aus dem Gesagten ergeben sich Folgen für eine Theorie der Demokratie als Revolte für verschiedene Teilgebiete der Demokratieforschung, die an der derzeitigen Krise der repräsentativen Demokratie ansetzen können. Die Demokratiequalitätsanalyse, die in den letzten Jahren einen gewissen Boom erlebt, kann von diesem Ansatz profitieren, indem die Revolte und ihre Bedingungen als notwendiges Demokratieprinzip anerkannt und als Indikatoren eingeführt werden und sie kann Aspekte von Bürgerschaft oder politischer Bildung berücksichtigen. Albert Camus' Werk erweist sich somit als nachhaltig und bietet eine Reihe politiktheoretischer Anknüpfungspunkte.

Die politischen Werke Camus' wurden in der akademischen Debatte bis dato kaum nachhaltig rezipiert oder gar weiterentwickelt. Es handelt sich hier um eine Lücke im Diskurs, den seine tiefgehenden Überlegungen zur menschlichen Existenz,

74 Vgl. Bauböck 2010.

75 Onfray 2013, S. 515.

76 Vgl. Interview mit dem Gründer der Hilfsorganisation Cap Anamur, Rupert Neudeck: http://syndikalismus.wordpress.com/2013/11/07/zum-100-geburtstag-von-albertcamus/ (Zugriff vom 25.11.2013) 
zu Freiheit und Gerechtigkeit sowie wie jener einer globalen Legislative oder einer bescheidenen Demokratie zu überbrücken in der Lage sind.

\section{Literatur}

Aronson, Raymond 2005. Camus \& Sartre. Amitié et Combat. Paris: Alvik Editions.

Aubéry, Pierre 1958. "Camus et la classe ouvrière", in French Review 23, 1, S. 14-21.

Alexander, Amy C.; Inglehart, Ronald; Welzel, Christian 2012. "Measuring effective democracy: a defense", in International Political Science Review 33, 1, S. 41-63.

Bartsch, Günter 1965. »Camus und der demokratische Sozialismus «, in Die Neue Gesellschaft 12, S. 787-792.

Bauböck, Rainer 2010. "Changing the boundaries of citizenship: the inclusion of immigrants in democratic polities", in Selected studies in international migration and immigrant incorporation, hrsg. v. Martiniello, Marco; Rath, Jan, S. 275-315. Amsterdam: Amsterdam University Press.

Brodocz, André; Schaal, Gary S. 2001. "Einleitung ", in Politische Theorien der Gegenwart II, hrsg. v. Brodocz, André; Schaal, Gary, S. 9-22. Opladen: Leske + Budrich.

Bruell, Cornelia; Mokre, Monika; Pausch, Markus. Hrsg. 2009. Democracy needs dispute. The debate on the European Constitution. Frankfurt a. M.: Campus.

Bühlmann, Marc et al. 2008. »Wie lässt sich Demokratie am besten messen? Zum Forumsbeitrag von Thomas Müller und Susanne Pickel ", in Politische Vierteljahresschrift 49, 1, S. 114-122.

Camus, Albert 1948. Réflexions sur une démocratie sans catéchisme, in La Gauche, Juillet 1948.

Camus, Albert 1950. Actuelles. Paris: Gallimard.

Camus, Albert 1957. Der Fremde. Düsseldorf: Karl Rauch Verlag.

Camus, Albert 1959 [1956]. Der Mythos von Sisyphos. Ein Versuch über das Absurde. Hamburg: Rowohlt.

Camus, Albert 1977 [1947]. Essais. Extrait de »Démocratie et Modestie«. Paris: Gallimard.

Camus, Albert 1989. Carnets III, mars 1951 - décembre 1959. Paris: Gallimard.

Camus, Albert 1997 [1953]. Der Mensch in der Revolte. Reinbek bei Hamburg: Rowohlt.

Camus 1997 [1960]. Fragen der Zeit. Reinbek bei Hamburg: Rowohlt.

Camus, Albert 1998 [1948]. Die Pest. Reinbek bei Hamburg: Rowohlt.

Crouch, Colin 2008. Postdemokratie. Frankfurt a. M.: Suhrkamp.

Dahrendorf, Ralf 2010 [1959]. Homo sociologicus: Ein Versuch zur Geschichte, Bedeutung und Kritik der Kategorie der sozialen Rolle, 17. Auflage. Wiesbaden: VS Verlag für Sozialwissenschaften.

Dewey, John 2008. The later works. Vol. 11: 1935-1937, hrsg. v. Boydston, Jo Ann. Carbondale: Southern Illinois University Press.

Downs, Anthony 1968. Ökonomische Theorie der Demokratie, hrsg. v. Wildenmann, Rudolf. Tübingen: J. C. B. Mohr.

Ginzburg, Carlo 1979. Der Käse und die Würmer: Die Welt eines Müllers um 1600. Frankfurt a. M.: Syndikat.

Guérin, Jeanyves 2009. Dictionnaire Albert Camus. Paris: Edition Robert Laffont.

Guérin, Jeanyves 2013. Albert Camus. Littérature et politique. Paris: Champions Classiques Essais.

Habermas, Jürgen 1968. Der Strukturwandel der Öffentlichkeit. Untersuchungen zu einer Kategorie der bürgerlichen Gesellschaft. Neuwied a. R.: Luchterhand.

Habermas, Jürgen 1992. Faktizität und Geltung. Beiträge zur Diskurstheorie des Rechts und des demokratischen Rechtsstaates. Frankfurt a. M.: Suhrkamp.

Hartmann, Martin; Offe, Claus. Hrsg. 2011. Politische Theorie und politische Philosophie. Ein Handbuch. München: C. H. Beck.

Hayden, Patrick 2013. »Albert Camus and rebellious cosmopolitanism in a divided world «, in Journal of International Political Theory 9, 2, S. 194-219.

Hessel, Stéphane 2011. Empört Euch! Berlin: Ullstein. 
Isaac, Jeffrey C. 1992. Arendt, Camus and modern rebellion. New Haven, London: Yale University Press.

Jaurès, Jean 2012 [1898]. "Socialisme et libéralisme. Extraits. Préface de Paul Magnette«, in Supplément offert par Philosophie Magazine 57.

Kaase, Max 2000. »Politische Beteiligung/Politische Partizipation «, in Handwörterbuch des politischen Systems der Bundesrepublik Deutschland, hrsg. v. Andersen, Uwe; Woyke, Wichard, S. 473-478. Wiesbaden: VS Verlag für Sozialwissenschaften.

Laclau, Ernesto; Mouffe, Chantal 1985. Hegemony and socialist strategy. Towards a radical democratic politics. London: Verso.

Lipset, Martin Seymour; Rokkan, Stein. Hrsg. 1967. Party systems and voter alignments. Crossnational perspectives. New York: The Free Press.

Magnette, Paul 2005. Citizenship. The history of an idea. Essex: ECPR Press.

Marin, Lou 1998. Ursprung der Revolte. Albert Camus und der Anarchismus. Berlin: Verlag Graswurzelrevolution.

Marin, Lou. Hrsg. 2013. Albert Camus: Libertäre Schriften (1948-1960). Hamburg: LaikaVerlag.

Meyer, Martin 2013. Albert Camus. Die Freiheit leben. München: Hanser Verlag.

Meyer, Thomas 2005. Die Zukunft der Sozialen Demokratie. Bonn: Friedrich-Ebert-Stiftung.

Onfray, Michel 2013. Im Namen der Freiheit. Leben und Philosophie des Albert Camus. München: Knaus.

Pateman, Carole 1970. Participation and democratic theory. London, New York: Cambridge University Press.

Pelinka, Anton 2011. »Bildung und Demokratie. Welche Bildungsziele braucht die Demokratie? «, in Zukunft. Bildung. Lebensqualität, hrsg. v. Popp, Reinhold; Pausch, Markus; Reinhardt, Ulrich, S. 153-166. Hamburg: LIT Verlag.

Proudhon, Pierre-Jospeh 2010 [1840]. What is property? An inquiry into the principle of right and of government. Auckland: Floating Press.

Rawls, John 2006 [1975]. Eine Theorie der Gerechtigkeit, hrsg. v. Höffe, Otfried. Berlin: Akademie Verlag.

Rey, Pierre-Louis 2006. »Le pari démocratique d'Albert Camus «, in Revue d'histoire littéraire de la France 106, 2, S. 271-284.

Sändig, Brigitte 2004. Albert Camus. Autonomie und Solidarität. Leipzig: Königshausen und Neumann.

Sartori, Giovanni 1997. Demokratietheorie. Darmstadt: Wissenschaftliche Buchgesellschaft.

Schmidt, Manfred G. 2000. Demokratietheorien, 3. Auflage. Opladen: Leske + Budrich.

Schumpeter, Joseph A. 1950. Kapitalismus, Sozialismus und Demokratie. Bern: Francke.

Thiery, Peter 2009. "Moderne politikwissenschaftliche Theorie ", in Politikwissenschaft. Eine Einführung, 6. Auflage, hrsg. v. Lauth, Hans-Joachim; Wagner, Christian, S. 194-232. Paderborn: Schöningh UTB.

Todd, Olivier 1999. Albert Camus. Ein Leben. Reinbek bei Hamburg. Rowohlt.

Watzlawick, Paul 2011. Man kann nicht nicht kommunizieren. Das Lesebuch, hrsg. v. Trunk, Trude. Bern: Huber Verlag. 
Zusammenfassung: Das Werk von Albert Camus wurde nach seinem Tod 1960 weitgehend auf seine literarische Dimension reduziert. Dass Camus ein politisches Oeuvre hinterlässt, das vielerlei Anknüpfungspunkte für die Politik- und insbesondere für die Demokratieforschung bietet, wurde aus mehreren Gründen lange Zeit in den Hintergrund gedrängt: Für die politische Linke war er ein Moralist, der sich mehr um metaphysische Fragen kümmerte als um historisch materialistische. Für die politische Rechte war er als Philosoph des Absurden und als ehemaliger Kommunist ohnehin indiskutabel. Und die politischen TheoretikerInnen nahmen ihn nicht als einen der ihren wahr. Dabei hätten sie sich zumindest von seinem Werk inspirieren lassen können, denn es liefert unter anderem Grundlagen für eine Theorie der Demokratie als Staatsform der Revolte. Aus dem Werk Albert Camus' lassen sich für die Demokratietheorie folgende Thesen ableiten: 1. Die Demokratie beruht auf der objektiven Absurdität unseres Daseins und auf der Unabschließbarkeit der Geschichte; 2. Die Demokratie ist die Staatsform der Revolte im Dienste der Freiheit und der Gerechtigkeit; 3. Die Demokratie ist die Staatsform des Zweifels und des Dialogs; 4. Die Demokratie ist die Staatsform der Fremdheit und der Solidarität.

Stichworte: Albert Camus, Demokratie, Revolte, Absurdität, Existenz, Demokratietheorie

\section{Democracy and rebellion. Albert Camus' relevance for political theory}

Summary: The political dimension of Albert Camus' writings has been neglected by political theory for a long time. Following his dispute with Jean-Paul Sartre about his book The Rebel, Camus was left aside and seen as an outsider among socialist thinkers. His oeuvre was increasingly reduced to its literary dimension. Only a few scientists have analyzed Camus' political writings, which indeed offer manifold links to democratic and political theory. The aim of this article is to show the relevance of his writings for current democracies by introducing the following theses: 1 . Democracy is based on the absurdity of our existence and the non-termination of history; 2. Democracy is the governmental form of revolt for the sake of freedom and justice; 3. Democracy is the governmental form of doubt and dialogue; 4. Democracy is the governmental form of foreignness and solidarity.

Keywords: Albert Camus, democracy, rebellion, absurdity, existence, democratic theory

Autor

Prof. (FH) Dr. Markus Pausch

Zentrum für Zukunftsstudien

Fachhochschule Salzburg

Urstein Süd 1

5412 Puch bei Salzburg

Österreich 